\title{
Diagnostic work-up in obstructive and inflammatory salivary gland disorders
}

\author{
Work-up diagnostico nella patologia ostruttiva e infiammatoria delle ghiandole \\ salivari

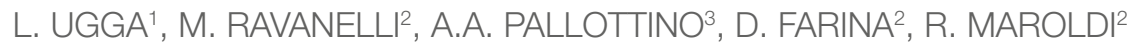 \\ ${ }^{1}$ Department of Advanced Biomedical Sciences, "Federico II" University, Naples, Italy; ${ }^{2}$ Department of Radiology, \\ University of Brescia, Brescia, Italy; ${ }^{3}$ Department of Radiology, Campus Biomedico University, Rome, Italy
}

\section{SUMMARY}

Inflammatory and obstructive disorders of the salivary glands are caused by very different pathological conditions affecting the gland tissue and/or the excretory system. The clinical setting is essential to address the appropriate diagnostic imaging work-up. According to history and physical examination, four main clinical scenarios can be recognised: (1) acute generalised swelling of major salivary glands; (2) acute swelling of a single major salivary gland; (3) chronic generalised swelling of major salivary glands, associated or not with "dry mouth"; (4) chronic or prolonged swelling of a single major salivary gland. The algorithm for imaging salivary glands depends on the scenario with which the patient presents to the clinician. Imaging is essential to confirm clinical diagnosis, define the extent of the disease and identify complications. Imaging techniques include ultrasound (US), computed tomography (CT) and magnetic resonance (MR) with MR sialography.

KEY WORDS: Salivary glands $\bullet$ Sialadenitis $\bullet$ Sialolithiasis $\bullet$ MRI $\bullet$ MR sialography

\section{RIASSUNTO}

La patologia infiammatoria ed ostruttiva delle ghiandole salivari riconosce molteplici eziologie con coinvolgimento del parenchima ghiandolare elo del sistema escretore. Il quadro clinico è essenziale per indirizzare l'integrazione diagnostica con adeguate metodiche di imaging. Sulla base dell'anamnesi e dell'esame obiettivo, possono riconoscersi quattro scenari clinici: (1) tumefazione acuta generalizzata delle ghiandole salivari maggiori; (2) tumefazione acuta di un'unica ghiandola salivare maggiore; (3) tumefazione cronica generalizzata delle ghiandole salivari maggiori associata o meno a xerostomia; (4) tumefazione cronica o persistente di una singola ghiandola salivare maggiore. L'algoritmo diagnostico per la scelta della metodica di imaging più appropriata dipende quindi dallo scenario clinico. L'imaging è essenziale per confermare la diagnosi clinica, per definire l'estensione della patologia ed identificare eventuali complicanze. Le metodiche di imaging disponibili includono l'ecografia, la tomografia computerizzata e la risonanza magnetica, anche con scialografia RM.

PAROLE CHIAVE: Ghiandole salivari $\bullet$ Scialoadenite $\bullet$ Scialolitiasi $\bullet$ MRI $\bullet$ Scialografia RM

Acta Otorhinolaryngol Ital 2017;37:83-93

\section{Introduction}

Inflammatory and obstructive disorders are more frequently observed in the major salivary glands (parotid, submandibular and sublingual) than in the small and scattered minor salivary glands. These disorders can be caused by very different conditions. This range can be simplified into two settings: the first includes diseases that target the gland tissue, while in the second the abnormality is mainly in the excretory system. An example of the first group is the interstitial infiltration of the gland as observed in acute viral infections or lymphoepithelial disease, whereas abnormalities in the composition (acute dehydration, previous irradiation) or the impaired drainage of the saliva (strictures, sialectasis, neoplastic compression of the main duct) are typical of the excretory system. Both the latter condi- tions may lead to the formation of calculi and facilitate bacterial infections. In summary, inflammation may be present without any excretory system obstruction, or it can lead itself to changes in the composition or drainage of saliva, a condition turning into acute or chronic inflammation. It is the clinical scenario that determines the appropriate diagnostic imaging workup, which includes studies such as ultrasound (US), CT, MR with MR sialography. US-guided fine-needle aspiration and cytology may be indicated to achieve correct diagnosis.

\section{Clinical scenarios}

A complete history and focused physical examination are crucial in evaluation of patients with obstructive/inflammatory salivary gland disorders. Key points should 
include the acute onset or long persistence of gland swelling, rate of change in the size of the gland (rapid or slow), associated fever or pain, drug exposures, history of malignancy and chronic illnesses such as rheumatologic diseases or sicca syndrome. Physical examination should include inspection of the face to observe swelling, induration, draining fistulae, scars from previous trauma, facial nerve paresis or paralysis, dryness or inflammation of the eye, and/or other masses. Careful inspection of the oral cavity should evaluate masses, swelling or lesions of the oral cavity, particularly in the Wharton's or Stensen's ducts area, and include massage (bimanually, when possible) of the gland to measure tenderness and determine if there is turbidity or purulence in the saliva, and assess the size and texture of the glandular tissue ${ }^{1}$. According to history and physical examination, four main clinical scenarios can be devised:

1. acute generalised swelling of parotid glands. Viral infection is the most probable cause. Less frequently the other major salivary glands are involved. Imaging is usually not indicated;

2. acute swelling of a single major salivary gland (pa$\mathrm{rotid} / \mathrm{submandibular}$ ). If associated with fever and pain (in some cases with a salivary colic), it raises the suspicion of sialolithiasis and/or acute suppurative sialadenitis. US is the first level imaging study indicated;

3. chronic generalised swelling of major salivary glands: several causes have to be considered. Bilateral painless and prolonged parotid swelling without signs of excretory abnormalities may indicate sialoadenosis (sialosis). It is a disorder usually associated with liver dysfunction, diabetes, or malnutrition ${ }^{2}$. If chronic diffuse swelling associated with "dry mouth" is observed, conditions leading to progressive parenchymal gland infiltration should be considered: Sjögren's syndrome, HIV sialadenitis and radiation-induced sialadenitis. In addition, systemic symptoms may be present in Sjögren's syndrome, sarcoidosis, IgG4-related disease and mycobacterial disease. The presence of adenopathies suggests chronic sialadenitis, including HIV-related and mycobacterial sialadenitis. US is indicated to assess changes both in the parenchymal echoic pattern and in the intra-extra-glandular excretory system;

4. chronic (prolonged) swelling of a single major salivary gland may be caused by (recurrent) sialolithiasis and sialadenitis. If unrelated to a previous confirmed diagnosis of sialolithiasis, a neoplastic cause has to be ruled out. A persistent soft and tender lump close to the gland may suggest a mucocele (ranula) or cystic lesion (branchial cyst, lymphatic malformation). Also in these conditions, US is indicated, and if necessary it can be combined with fine needle aspiration.

The algorithm for imaging the salivary glands depends on the scenario with which the patient presents to the clinician ${ }^{3}$. In most conditions, US is the first test. Crosssectional imaging is indicated to obtain a panoramic view and a better characterisation. In the setting of a patient with acute sialadenitis and suspicion of extra-glandular complications such as a neck abscess, contrast-enhanced CT is the modality chosen due to its high spatial resolution, the possibility to detect calcified stones and fast acquisition time. In chronic disorders, MR is the best imaging modality to study major and minor salivary glands due to its higher contrast resolution, multiparametric evaluation and submillimetric spatial resolution. Moreover, MR sialography has almost fully replaced conventional sialography in clinical practice.

\section{Imaging modalities}

\section{Conventional sialography}

In conventional sialography, the injection of a positive contrast medium into the opening of Stensen/Wharton's ducts reveals the anatomy of the main duct and intraglandular branches. Nowadays, sialography is infrequently employed because of the progressive improvements in CT and MR imaging, as well as changes in the clinical management of major salivary gland non-neoplastic disease.

\section{Ultrasound}

Ultrasound is a useful technique for detection of nodules of the parotid and submandibular glands. The examination should be carried out with the highest-frequency transducer possible to permit accurate evaluation of the nodule and/or the parenchyma ${ }^{4}$. The deep portions of the parotid gland require the use of less detailed lower-frequency linear probes $(5-7 \mathrm{MHz})$, given the need for deeper penetration. Nevertheless, the deep part of the gland, close to the stylomandibular notch, or the parapharyngeal space are difficult to image, frequently hidden by the mandible. For lesions located/extended into the deep parotid gland, cross-sectional imaging is recommended ${ }^{5}$.

\section{$C T$}

CT is the preferred imaging modality for acute sialadenitis given its spatial resolution and sensitivity to calcification. Limitations of CT include extensive dental artifacts that cannot be avoided with angled axial imaging, contraindications to the use of contrast agent and radiation concerns in the paediatric population. Iodinated contrast intravenous administration is helpful for evaluation of lesion enhancement, assessment of lymph nodes, analysis of the extent of inflammation in infections, and for understanding the vascularity of lesions ${ }^{5}$. Contiguous, axial, thin 2-mm slices are typically acquired from the skull base to the hyoid bone and 
the images are reformatted at $1 \mathrm{~mm}$ for display. On CT systems where multiplanar reformats are not possible, axial images should be acquired parallel to the occlusal plane of the maxilla or the inferior border of the mandible to best visualise the course of Stensen's duct or Wharton's duct. Multiplanar reformats are performed in the coronal and sagittal plane ${ }^{6}$.

\section{$M R$ and MR sialography}

The superior tissue contrast with MR permits excellent discrimination of the parenchyma and ductal structures. The use of fluid-sensitive pulse sequences allows for non-invasive sialography. Most MR sequences are obtained with a $3 \mathrm{~mm}$ slice thickness and $0.5 \mathrm{~mm}$ to $2 \mathrm{~mm}$ interslice gaps with a multi-channel head coil. Superficial coils may be used to achieve a better spatial resolution for superficial structures. A recent study demonstrated that high resolution MR imaging with a microscopy coil can readily delineate the labial glands ${ }^{7}$. T1-weighted imaging allows better tissue contrast in parotid glands because of the presence of fat tissue within the stroma, yielding a high T1 signal. T2-weighted and post-contrast fat-suppressed T1-weighting imaging are also recommended. Because fat also has high T2 signal on fast spin echo imaging, fat suppression on $\mathrm{T} 2$ weighted images helps to generate tissue specificity. Axial and coronal views are generally obtained. Nonenhanced T1-weighted images combined with nonfat-suppressed fast spin-echo T2-weighted images are optimal for delineation of lesions and prediction of the nature of parotid gland pathology. The administration of a paramagnetic contrast agent is helpful to distinguish between solid and cystic components. Contrast administration also helps to evaluate the margins of the mass and its extension into surrounding tissue planes. Diffusion-weighted MR imaging, quantified by apparent diffusion coefficient (ADC), evaluates the diffusion of water molecules. A study of normal and diseased salivary glands with ADC mapping showed that ADC values increased in sialadenitis and decreased in abscess. These changes in ADC may be due to changes in the extracellular water content and its viscosity in the gland parenchyma ${ }^{8}$. MR sialography is a noninvasive method to characterise the ductal structure of the parotid and submandibular glands, providing an excellent alternative to conventional sialography. It is performed with a fat-suppressed heavily T2-weighted high-resolution fast spin echo sequence with a surface coil or multichannel head coil. Several studies have demonstrated that MR sialography is generally as accurate as conventional sialography in detecting obstructions, stenosis and stricture of the main ducts, but it is limited by the acquisition time required for a single sequence and susceptibility to motion 569 .

\section{Infectious sialadenitis}

\section{Acute suppurative sialadenitis and salivary gland emergencies}

Acute suppurative sialadenitis is a bacterial infection characterised by the sudden onset of painful unilateral, occasionally bilateral, parotid swelling with the submandibular salivary gland infrequently involved; it is usually related to inflammation or ductal obstruction. Retrograde non-obstructive infections are more frequent in the parotid glands. Obstructive sialadenitis is usually observed in the submandibular glands, most commonly due to calculi and possibly complicated by bacterial infection. Usually imaging is not necessary at initial presentation. However, if there is no improvement after 48 hours, imaging is recommended to identify/rule out an abscess or an obstructive process such as sialolithiasis. Imaging is also indicated when sepsis is suspected, or if the diagnosis is uncertain. Imaging options include CT, MR sialography, or US. If sialolithiasis with secondary sialadenitis is suspected, US is indicated as it detects up to $90 \%$ of stones $2 \mathrm{~mm}$ or greater. With CT, thin slices must be acquired to better identify very small calculi. When treatment planning requires demonstrating the calculus (or stricture) and overall excretory system, MR sialography is indicated. MR sialography does not require intraductal injection of contrast agent. It assesses the overall ductal architecture of the gland. It is possibly superior to US in stone detection, but is limited by time requirements. This limitation hampers its wide use. In sialadenitis, inflammatory changes of major salivary gland limited to the parenchyma can be adequately shown by US. If extra-glandular extension is suspected, contrast-enhanced CT is the most sensitive tool for detecting and mapping the deep extent of an abscess. On CT imaging and also on MR, initially the inflamed gland appears enlarged, shows abnormal high attenuation or T2 intensity and a relevant post-contrast enhancement ${ }^{10}$. Signs to be scrutinised include thickening of fascia and infiltration of subcutaneous fat. Thickening of deep cervical fascia and infiltration of cervical fat produce a "dirty fat" appearance. Cellulitis of the gland can lead to formation of a focal abscess ${ }^{11}$ (Figs. 1-4). Reconstructions of the CT volume in the coronal plane are indispensable to evaluate the extent and topographic relationship of cellulitis or abscess (floor of the mouth for submandibular lesions and skull base for parotid gland) ${ }^{10}$. The mortality for suppurative parotitis has improved dramatically in the era of antibiotics, but is still reported to be $20 \%$ to $40 \%$. Potential complications are rare, but can be serious. These include abscess extension into deep spaces of the neck and mediastinum, septic thrombophlebitis of the jugular vein (Lemierre's syndrome), osteomyelitis of the mandible, sepsis, respiratory obstruction and rupture through the external auditory canal with spontaneous drainage through the face ${ }^{12} 13$. 


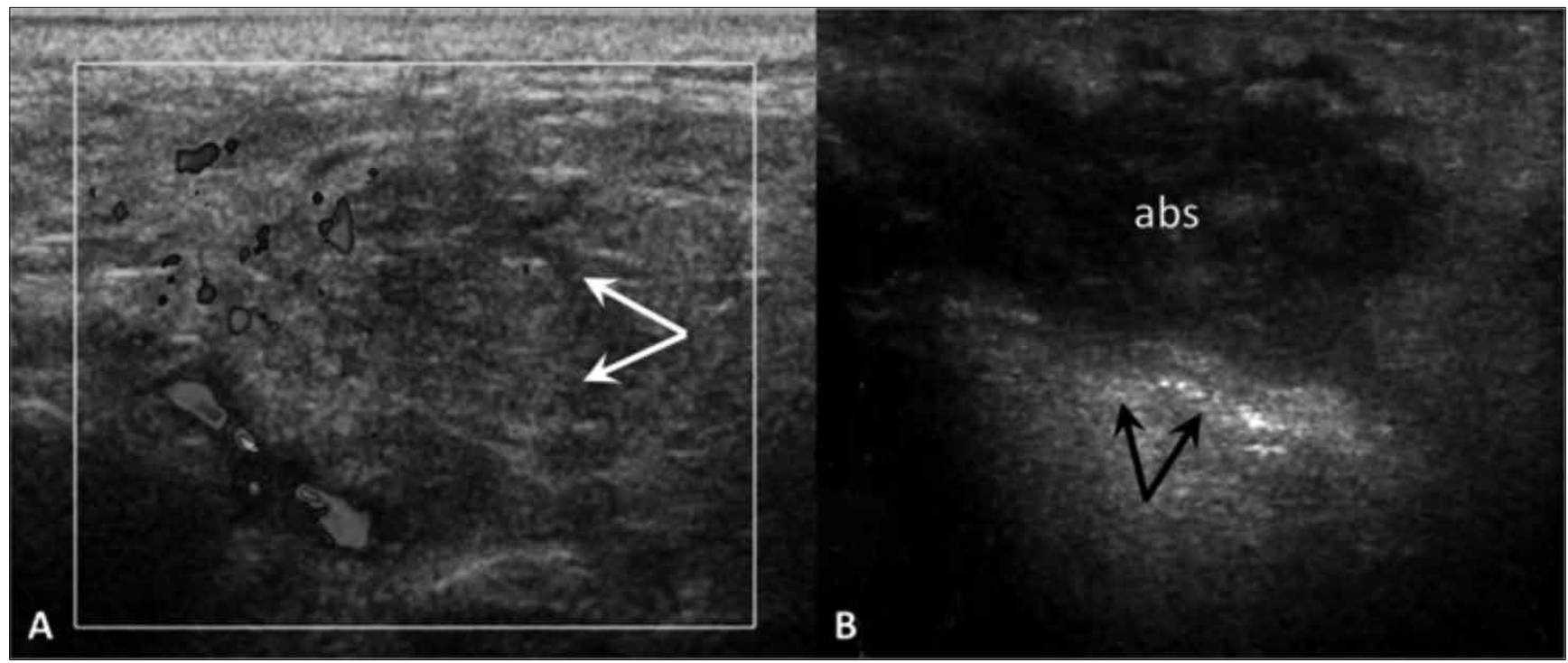

Fig. 1. Acute parotid sialadenitis (A). Enlarged, hypoechoic parotid gland (arrows), colour Doppler shows increased blood flow. Parotid gland abscess (B). US demonstrates a heterogeneous hypoechoic collection (abs) within the parotid gland: internal echoes, poorly defined borders and posterior acoustic enhancement (arrows).

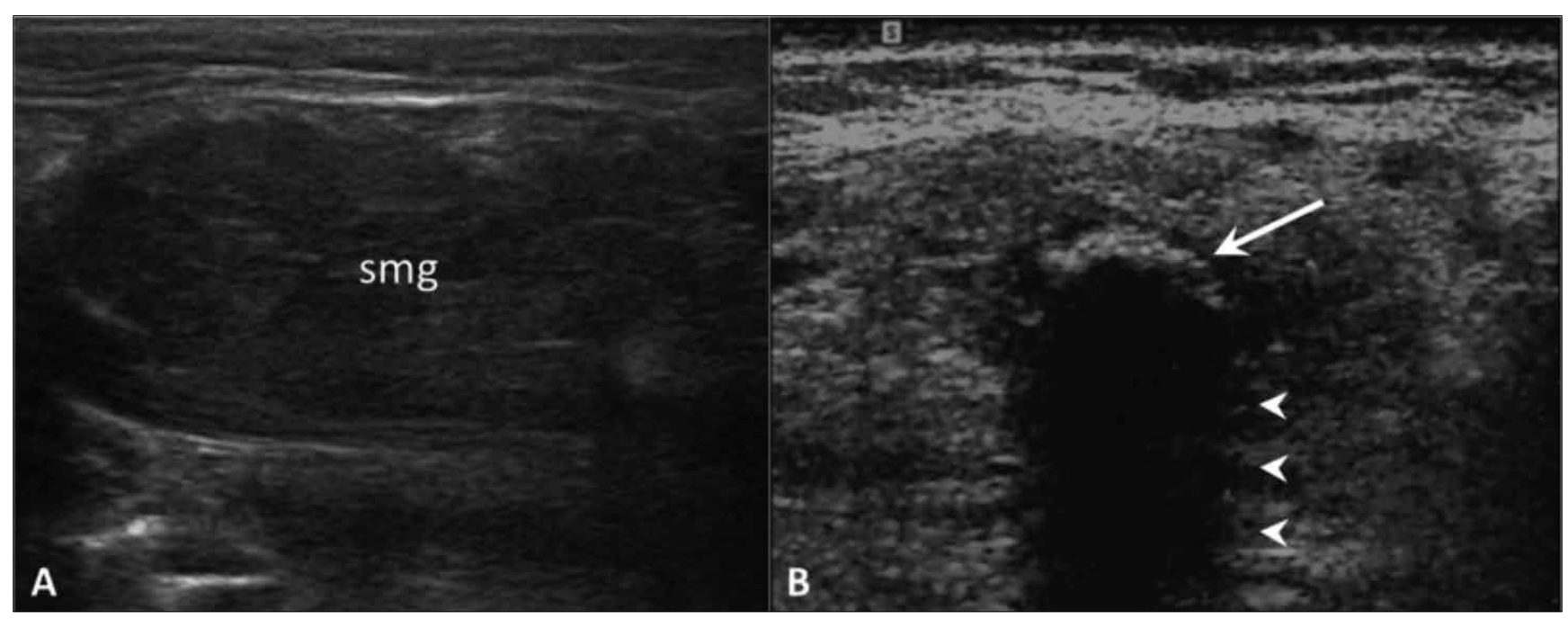

Fig. 2. Obstructive sialadenitis. On US, the submandibular gland (smg) appears enlarged and hypoechoic (A). Highly reflective echogenic focus within the Wharton's duct (arrow) with prominent posterior acoustic shadowing (arrowheads), consistent with a sialolith (B).

\section{Tuberculosis}

Tuberculous (TB) sialadenitis with primary or secondary manifestations in the lymph nodes of the parotid gland is more frequently observed in developing countries. However, in the last few years, non-tuberculous mycobacteria are more often diagnosed. Particularly in children with enlarging, non-tender mass with violaceous skin discolouration, unresponsive to conventional antibiotics, this diagnosis must be taken into consideration. The diagnosis may be suggested by US when enlarged intra-parotid lymph nodes and a diffusively enlarged parotid gland are demonstrated. Contrast-enhanced CT most commonly shows asymmetric cervical lymphadenopathy and contiguous low-density, necrotic, ring-enhancing masses in- volving the subcutaneous fat and skin ${ }^{14}$. TB sialadenitis more often affects the parotid gland (70\%) where two different clinical presentations are usually observed: one is like acute sialadenitis, with imaging features resembling acute sialadenitis with abscess; the other mimics a salivary gland tumour. In this setting, it is necessary to rule out other conditions presenting with single gland enlargement combined with abnormal lymph nodes such as lymphoma or metastatic disease ${ }^{15}$.

\section{Viral sialadenitis}

Several viruses have been associated with sialadenitis. Among these, the paramyxovirus (mumps) is the best known of the sialoadenotropic viruses. On US, 


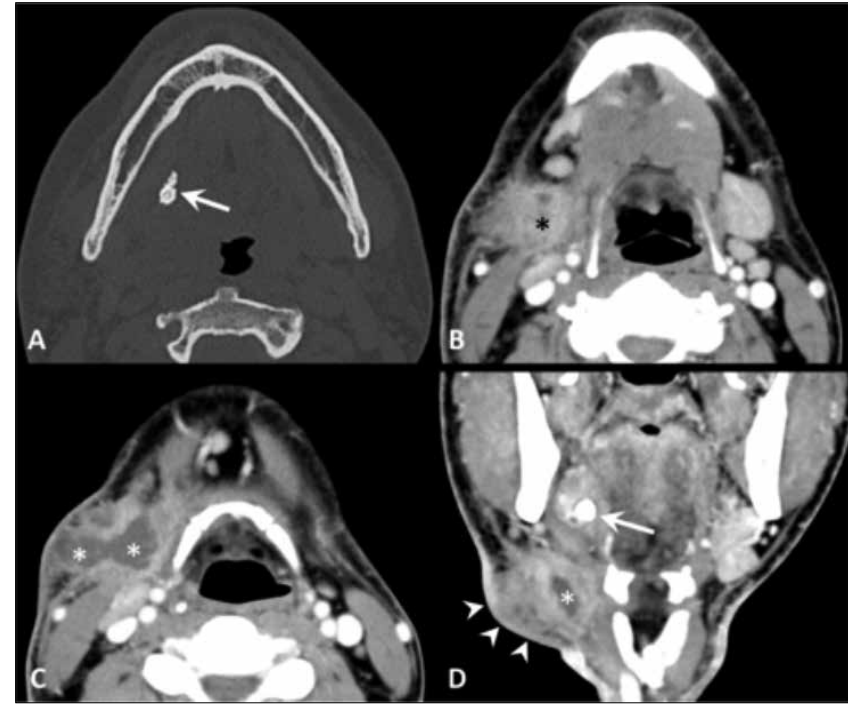

Fig. 3. Obstructive sialadenitis complicated by extra-glandular abscess, contrast-enhanced CT. (A) The bone window image demonstrates a mineralised stone (arrow) within the right Wharton's duct. (B-D) The soft tissue window images show enlargement of the right submandibular gland (black asterisk), thickening of the submandibular fat tissue producing a "dirty fat" appearance. At the level of the neck swelling (arrowheads), CT demonstrates an abscess (white asterisks) in the submandibular space. The calculus is shown also the coronal plane (arrow).

the glands are enlarged with a more rounded shape, a convex lateral surface and a hypoechoic structure. CT and MR are not indicated and rarely performed in acute viral sialadenitis. On CT or MR images, the inflamed glands appear enlarged and may show abnormal (hyperdense) attenuation or intensity (high-signal intensity on $\mathrm{T} 2$ weighted images), enhancing after administration of contrast agent.

\section{Human immunodeficiency virus}

Persistent, painless parotid enlargement, usually bilateral, occurs in about $5 \%$ of HIV-positive patients. As the disease progresses, the lymphoproliferative activity of parotid lymph nodes or proliferation of lymphocytes that are normally present within the gland or have infiltrated into the gland will result in an enlarged parotid gland. Bilateral benign parotid lymphoepithelial cysts can also develop and cause progressive glandular swellings. These cysts are thought to originate from included epithelium in the intraparotid lymph nodes ${ }^{2}$. US demonstrates large anechoic/hypoechoic areas with debris and septa (lymphoepithelial cysts) combined with large oval hypoechoic areas (enlarged intraparotid nodes). Benign lympoepithelial lesions and cysts have non-specific CT and MR features (Fig. 5). In these patients, additional abnormalities in the Waldeyer ring (tonsillar hypertrophy) and reactive cervical adenopathy are frequently present ${ }^{16} 17$.

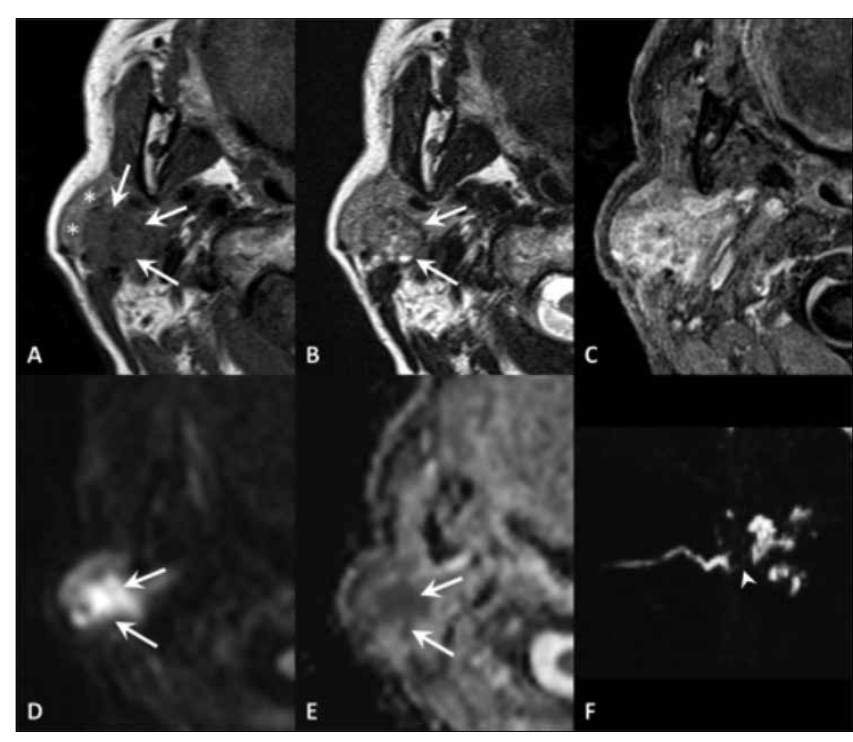

Fig. 4. Focal obstructive sialadenitis. MR axial SE T1 (A), TSE T2 (B), VIBE after contrast administration (C), DWI b1000 (D) with ADC map (E); sialo-MR (maximum intensity projection in the sagittal plane) (F). Sudden onset of painful swelling of the right parotid gland. The focal inflamed area (arrows) can be detected because of its mass-effect on the adjacent gland tissue (asterisks), greater post-contrast enhancement and diffusion restriction. The MR sialography shows a filling defect (arrowhead) and intraglandular duct ectasia.

\section{Chronic sialadenitis and related conditions}

\section{Sjögren's syndrome and lymphoepithelial sialadenitis}

Sjögren's syndrome is a multisystem autoimmune disease, mostly affecting women. It is characterised by a diffuse lymphoid proliferation in the exocrine salivary and lacrimal glands leading to lymphocytic sialadenitis, parenchymal replacement and progressive dilatation of intraparenchymal ducts. MR and US are more accurate than CT in assessing the severity of the disease. In the early stage of the disease, MR shows only enlarged and homogenous parotid glands. As the disease progresses, tiny ductal dilatation and parenchymal lymphocytic infiltration result in a non-homogeneous dotted or nodular pattern ("salt and pepper"). This corresponds to intermediate disease. In this stage, US shows dilated ducts and lymphoproliferative lesions as a pattern of anechoic and hypoechoic scattered areas. On CT, the accurate scrutiny of thin slices may show multiple hypodense "spots" reflecting duct dilations ${ }^{18}$. The advanced stages are characterised by marked non-homogeneous appearance of the glands: large dilated intraglandular ducts (foci of high T2 signals) are combined with an overall decrease in T2 signal (focal accumulation of lymphocytes and fibrous tissue). This combination gives rise to a "honeycomb" pattern ${ }^{11}$ (Fig. 6).

The progressive changes in size of intraglandular ducts have been evaluated with high resolution MR sialography. An equivalent of the sialographic staging of Sjögren's 


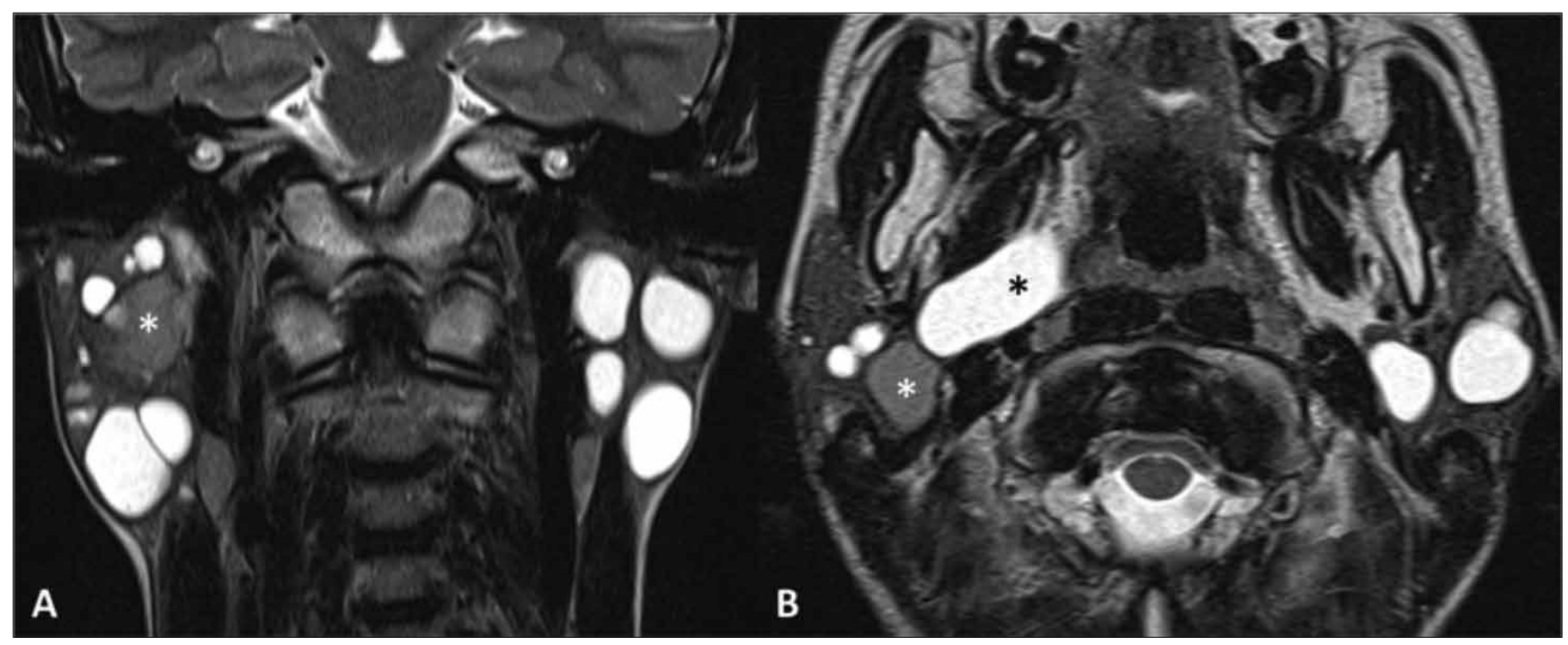

Fig. 5. Bilateral benign parotid lymphoepithelial cysts in a HIV-positive patient. MR TSE T2-weighted sequences on the coronal (A) and axial (B) planes. Multiple cystic lesions in the parotid glands, among which a high-protein content cyst (white asterisk) and a cyst extending in the right parapharyngeal space reaching the lateral wall of oropharynx.

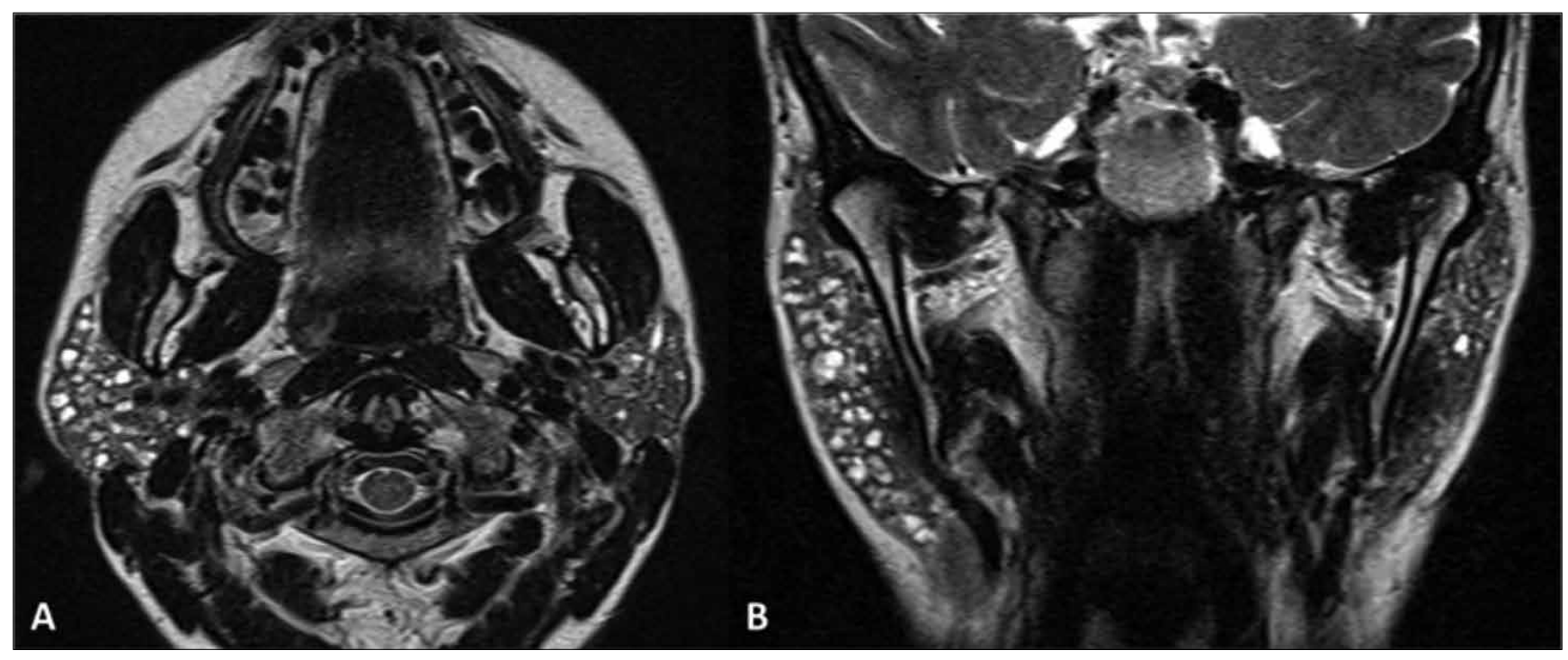

Fig. 6. Sjögren's syndrome - intermediate disease. MR TSE T2-weighted sequences on the axial (A) and coronal (B) planes. Bilateral parotid tiny ductal dilatation, particularly evident on the right.

syndrome described by Rubin and Holt in 1957 is now applied to MR sialography, as follows ${ }^{17}{ }^{19}$ :

- Stage I. Punctate contrast collection $1 \mathrm{~mm}$;

- Stage II. Globular contrast collection 1 to $2 \mathrm{~mm}$;

- Stage III. Cavitatory contrast collection $2 \mathrm{~mm}$;

- Stage IV. Destruction of gland parenchyma.

Makula et al. consider MR imaging to be unnecessary as a routine method in the diagnosis of Sjögren's syndrome. They consider US adequate for both the diagnosis and the follow-up ${ }^{20}$.

Patients with primary Sjögren's syndrome have an increased risk of non-Hodgkin's lymphoma, particularly extranodal marginal zone B-cell lymphoma of mucosa- associated lymphoid tissue (MALT) type, most of which occur in the salivary glands (Fig. 7).

\section{Sarcoidosis}

Parotid gland involvement is demonstrated in 6\%-30\% of patients with sarcoidosis. On MR images, the affected parotid glands are typically enlarged, with increased signal intensity on T2-weighted images and enhancement on contrast-enhanced images ${ }^{21}$. Large granulomas have been described as multiple benign-appearing, non-cavitating, intraparotid nodules ("foamy parotids") ${ }^{22}$. Diagnosis is established when clinical and imaging findings are integrated with histology ${ }^{24}$. 


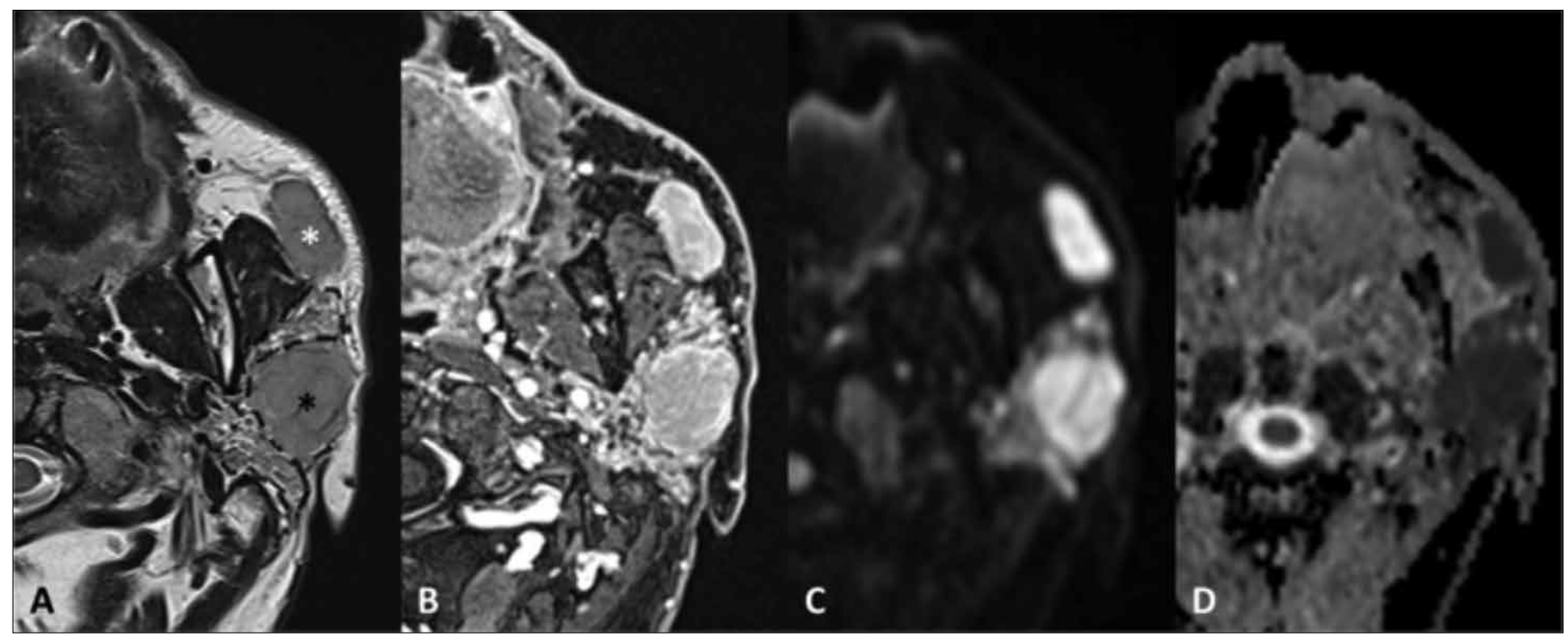

Fig. 7. Lymphoma of mucosa-associated lymphoid tissue (MALT) in a patient with primary Sjögren's syndrome. MR TSE T2 (A), VIBE after contrast administration (B), DWI b1000 (C) with ADC map (D). MR scan shows a lesion of the superficial portion of the parotid gland (black asterisk), displacing the remaining part of the gland (dashed line), with enhancement and high diffusion restriction. A second lesion with similar features is evident at the anterior extension of the gland (white asterisk).

\section{Radiation-induced sialadenitis}

Radiation-induced sialadenitis is a sequela of external beam radiation or radioactive iodine therapy for thyroid carcinoma. Combining medical history with clinical examination facilitates a diagnosis of hyposalivation due to radiation sialadenitis, and imaging is not required. The pathologic changes are characterised by a combination of patches of inflammatory infiltrates mixed with areas of fibrosis and volume reduction. It results in a marked heterogeneous appearance ${ }^{2324}$. On US, these structural changes translate into hypo- or isoechoic areas (relative to adjacent muscles) with multiple hyperechoic lines or spots. On MR, the early post-RT changes are characterised by enlarged glands with hyperintensity on T2-weighted images and marked enhancement. At later stages, the signal intensity of the glands decreases while the volume constantly reduces, leading to gland shrinking, and the enhancement also diminishes. MR sialography demonstrates a reduced visibility of the ducts in the salivary glands that received more than $20 \mathrm{~Gy}{ }^{23}$.

\section{IgG4-related disease}

In the head and neck, the salivary glands are the most frequently involved anatomic structures by IgG4-related disease, with the submandibular gland being the most common target. Chronic sclerosing sialadenitis (Kuttner tumour) and Mikulicz's disease are now considered to be part of the spectrum of IgG4-related disease. Kuttner tumour typically affects one or both submandibular glands and clinically presents as a "hard swelling". US shows diffuse enlargement of the submandibular glands with multiple ill-defined hypoechoic foci scattered against a heterogeneous background giving rise to "mottled, netlike" appearance and minimally increased vascularity.
Enlarged glands show homogeneous attenuation on CT and low to intermediate signal intensity on $\mathrm{T} 2$-weighted images and intermediate signal intensity on T1-weighted images on MR imaging with associated homogeneous enhancement. Multiple enlarged lymph nodes are commonly seen. Differential diagnosis includes malignant salivary gland tumours, if unilateral involvement is seen; lymphoma and the acute phase of Sjögren's syndrome, in case of bilateral lesions. Mikulicz's disease is clinically defined as bilateral painless swelling of the submandibular, sublingual, parotid and lacrimal glands for a duration of at least 3 months. Cross sectional imaging shows bilateral symmetric swelling of the involved glands. At CT, lesions usually demonstrate homogeneous attenuation and enhancement. On MR imaging, lesions typically demonstrate a homogeneous low to intermediate signal intensity on T2-weighted images and low signal intensity on T1weighted images, with homogeneous enhancement following contrast agent administration. Differential lesions to be considered include the acute phase of Sjögren's, lymphoma, sarcoidosis and mumps 2526 .

\section{Sialolithiasis}

Sialolithiasis is the main cause of obstructive salivary diseases, being involved in $66 \%$ of cases and accounting for about $50 \%$ of major salivary gland diseases. Sialolithiasis affects the submandibular gland in $80-90 \%$ of cases, mainly unilaterally, without a preferred side. This different incidence of calculi between the parotid and submandibular gland is partially related to the ascendant and sharper angled duct system of the submandibular gland and the type of secretion, mainly mucous. The sublingual and other minor salivary glands are rarely affected. 
Currently, US represents an excellent first-level diagnostic technique insofar as, in experienced hands, it reveals ductal and highly mineralised stones with a diameter of at least $1.5-3 \mathrm{~mm}^{27}$. Because of this size-related threshold, US does not allow a reliable exclusion of very small salivary gland calculi. Therefore, further diagnostic investigations are recommended to detect calculi in patients with normal US findings and suspected lithiasis ${ }^{28}$. In the workup, it is important to differentiate calculi located in the main ducts from those placed in the intraglandular ducts, as their treatment may require removal of the gland ${ }^{29}$. Sonographically, intraductal concretions appear as bright curvilinear echo complexes with a posterior shadowing. In calculi smaller than $2 \mathrm{~mm}$, the shadow may be missing. In symptomatic sialolithiasis, a concomitant dilatation of the ductal system or inflammation is often visualised ${ }^{29}$. $\mathrm{CT}$, MRI and MR sialography can be reserved to patients with negative or inconclusive US results and a clinical presentation suggesting ductal obstruction. An important advantage of MR sialography is the fact that the structural anatomy of the salivary glands remains unchanged with this technique, which allows an exact delimitation of the glandular parenchyma and duct. In US, the parenchyma and ducts may be compressed by the transducer. MR sialography consists of two steps: an anatomical study, which contributes to define the dimensional, morphological and structural features of the parotid and submandibular glands and the relationship with adjacent structures; and a sialographic study, which represents the ductal components and possible intraductal filling defects. In addition, it offers simultaneous assessment of both the parotid and submandibular glands even in acute inflammation ${ }^{930}$ (Figs. 8, 9).

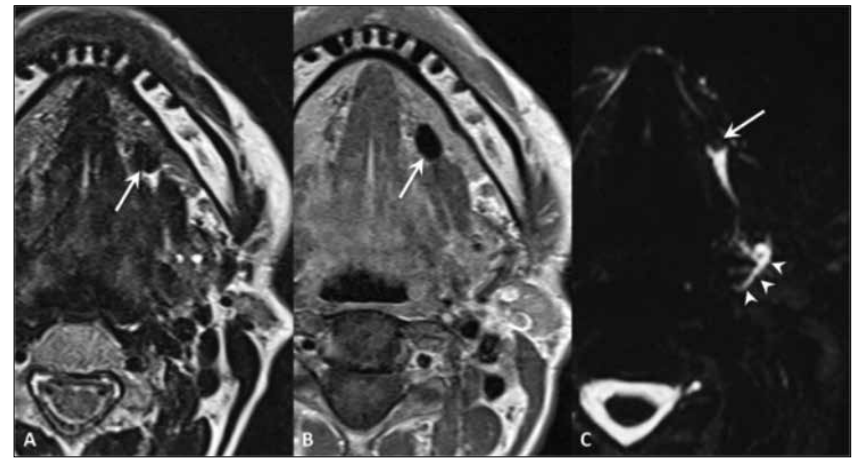

Fig. 8. Wharton's duct sialolithiasis. MR TSE T2 (A), SE T1 (B) and MR sialography (maximum intensity projection in the axial plane) (C). Hypointense mineralised stone in the left Wharton's duct, to which corresponds a filling defect on sialo-MR (arrows); moderate ducts dilatation (arrowheads).

\section{Sialadenosis}

Sialadenosis, or sialosis, is defined as a bilateral persistent, painless, soft, non-neoplastic, non-inflammatory swelling usually involving both parotid glands and at times the submandibular salivary glands. Sialadenosis is associated with a variety of conditions that include alcoholism, endocrine disorders (particularly diabetes mellitus) and malnutrition, which in our society usually results from anorexia nervosa ${ }^{2}$. Diagnosis is best achieved by integrating the patient's medical history, clinical signs and symptoms of glandular swelling and information derived from available investigative procedures that show bilateral enlargement of the parotid glands with fatty infiltration in the end stage of disease ${ }^{18}$.

\section{Cystic lesions}

Cystic lesions may occur in both minor and major salivary glands; their size is usually related to the site of ori-

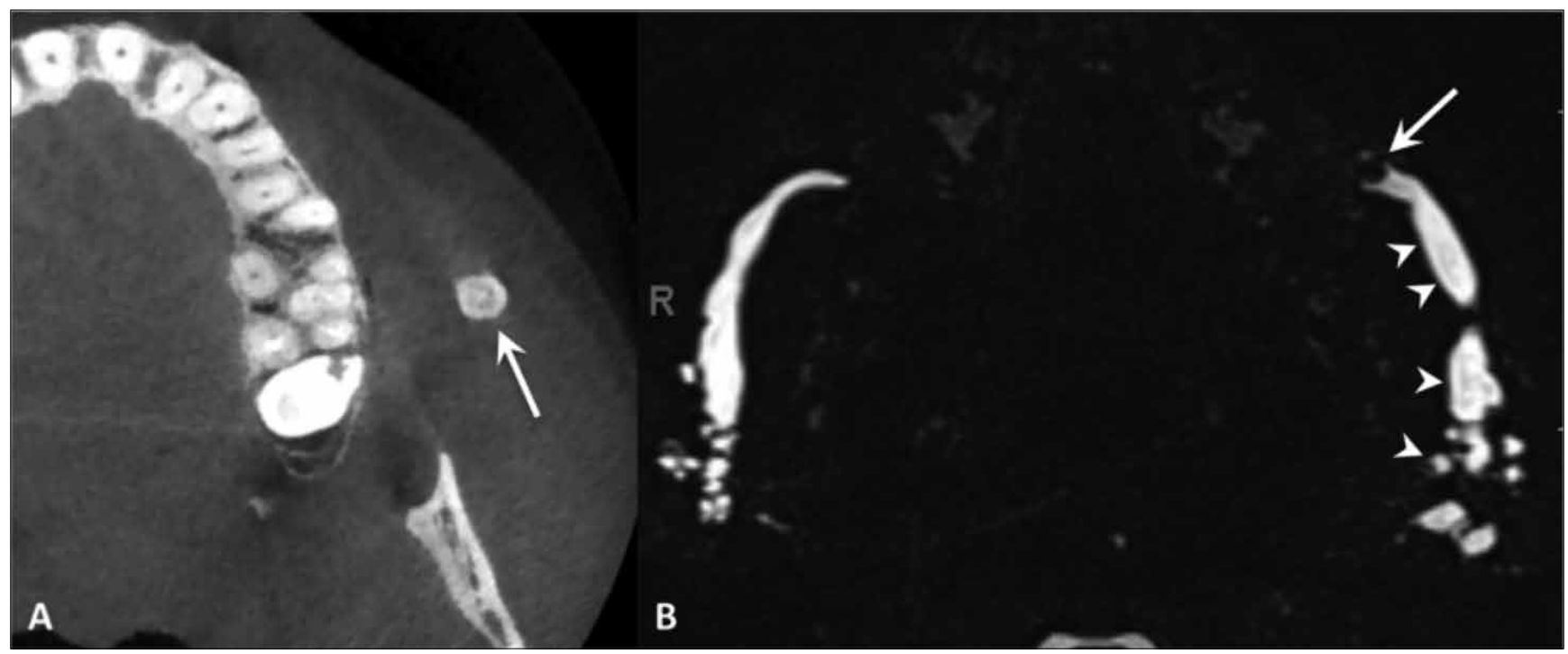

Fig. 9. Stensen's duct sialolithiasis. Cone-beam CT (A) and MR sialography (maximum intensity projection in the axial plane) (B). Highly mineralised stone in the left Stensen's duct, corresponding to a filling defect on MR sialography (arrow) causing a marked dilatation of the ductal system (arrowheads); dilatation of the contralateral ductal system is also evident. 
gin, reaching greatest dimensions in major salivary glands. Mucous retention cysts may develop consequent to a duct obstruction, representing true cysts, generally unilocular, with epithelial linings. Conversely, a mucocele is a benign, mucus-containing pseudocystic lesion caused by a mucus extravasation. The common site of occurrence of mucocele is the lower lip, followed by tongue, floor of mouth (ranula), and the buccal mucosa. When the mucocele content becomes infected, the lesion is defined as mucopyocele (Fig. 10). Sublingual glands are more at risk for duct obstructions because of the small caliber of the drainage ducts.

\section{Ranula}

The term ranula refers to a mucous retention cyst that occurs primarily in the sublingual gland. A ranula can occur in two forms: simple ranula, the most common, is a retention cyst that remains above the level of the mylohyoid muscle and deep or plunging ranula, which represents a submucosal mucus extravasation phenomenon (pseudocyst) that develops as a leakage from a sublingual duct.

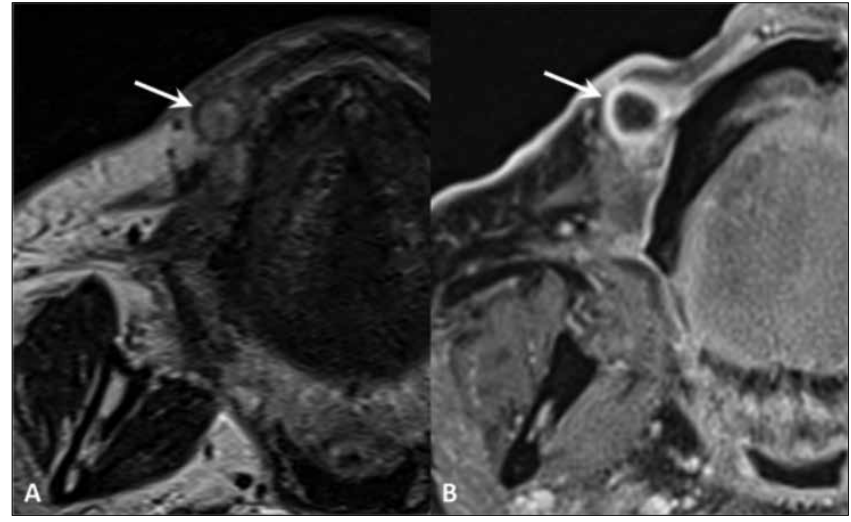

Fig. 10. Mucopyocele. MR TSE T2 (A) and post-contrast VIBE (B). Spherical lesion within the soft tissues of the lip with ring-enhancement and hypointensity on T2, corresponding to a mucopyocele of a labial gland (arrow).

Secretory leakage occurs when the duct wall is lacerated, probably from trauma. The rapid accumulation of fluid is cyst-like in appearance and presents itself as either an intraoral or extraoral swelling. Plunging ranula may extend

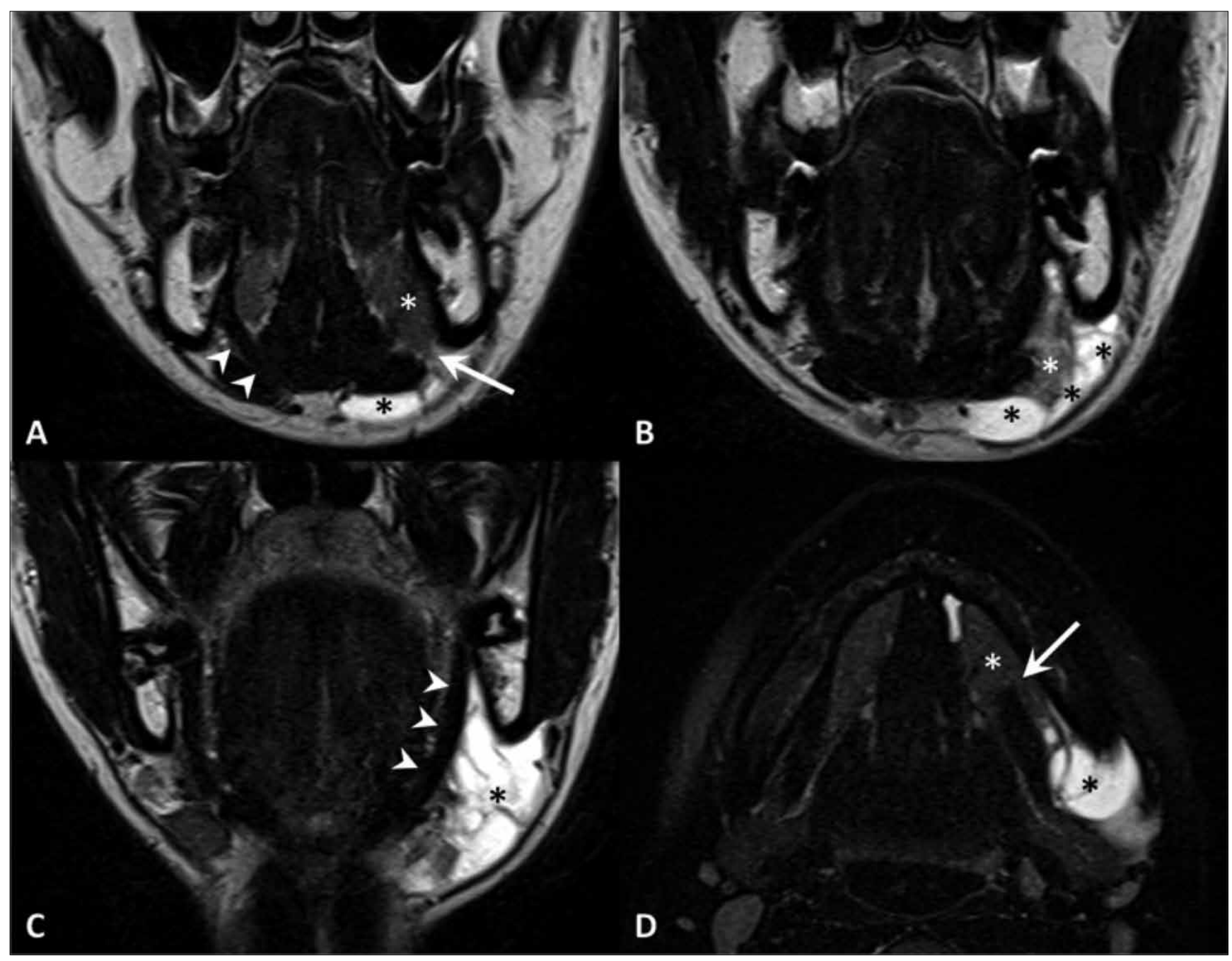

Fig. 11. Plunging ranula. TSE T2, coronal plane (A-C, anterior to posterior), TSE T2 with fat-saturation, axial plane (D). MR shows a defect of the mylohyoid muscle (arrow) with sublingual gland (white asterisk) herniation. Note the normal appearance of the contralateral and ipsilateral (in a posterior slice) mylohyoid muscle (arrowheads). Plunging ranula (black asterisks) extends posteriorly in the submandibular space. 
through the mylohyoid muscle and deeper soft tissue to produce a submental or lateral neck swelling termed plunging or cervical ranula. The plunging ranula often infiltrates adjacent tissue planes, extending inferiorly and dorsally to the submandibular gland region, while ventrally it may cross the midline to the contralateral floor of the mouth. US is very useful in determining the extent of the plunging ranula, confirming the cystic nature of the lesion, assessing the status of the mylohyoid muscle (a defect demonstrated in nearly $100 \%$ of cases) and evaluating the sublingual gland for rupture or herniation ${ }^{31}$. On CT, the simple ranula is usually a roughly ovoid-shaped cyst with a homogeneous central attenuation region of 10 to $20 \mathrm{HU}$. On MR imaging, a ranula's characteristic appearance is generally dominated by its high water content. Thus, it has a low T1-weighted, an intermediate proton density, and a high T2-weighted signal intensity. This appearance, especially in a plunging ranula, may resemble that of a lymphatic malformation (Fig. 11).

\section{Branchial cleft cysts}

First branchial cleft cysts are usually periauricular or periparotid/intraparotid. These latter cysts/sinus tracts are classified as type II and are the most common first branchial cleft cyst. On US, they are usually solitary anechoic masses with posterior acoustic shadowing. On CT imaging, first branchial cleft anomalies usually present as a cystic mass superficial/within/deep to the parotid salivary gland ${ }^{32}$. They are hyperintense on T2-weighted imaging and hypointense on T1-weighted imaging. As with other branchial cleft anomalies, cyst wall thickness and enhancement varies with the degree of inflammation. In contrast, a second branchial cleft cyst is often seen deep to the platysma, anterior to the sternocleidomastoid muscle, posterior to the submandibular gland, and lateral to the carotid sheath. It often lies inferiorly to the parotid gland ${ }^{5}$.

\section{Tumour-like lesions}

\section{Necrotising sialometaplasia}

Necrotising sialometaplasia is a benign, ulcerative, selflimiting inflammatory disease of the minor salivary glands. It probably results from a compromised vasculature supplying salivary gland tissue. The palatine salivary glands represent the most frequent group involved ${ }^{33}$. On MRI, it presents as a lobulated submucosal lesion of the hard palate, hyperintense on T2 and hypointense on T1 weighted images; after contrast administration, a thin peripheral rim of enhancement may be observed. CT demonstrates the absence of bone erosion ${ }^{34}$.

\section{Conclusions}

Obstructive and inflammatory disorders of the salivary glands are commonly seen in the major salivary glands due to a various pathological conditions, affecting both the glandular tissue and/o the escretory system.

The appropriate diagnostic imaging workout is determined by the clinical scenario which is essential to narrow the list of differential diagnosis.

US-guided fine needle aspiration and cytology may also attribute to achieve the correct diagnosis.

\section{References}

1 Blitzer A. Inflammatory and obstructive disorders of salivary glands. J Dent Res 1987;66 Spec No:675-9.

2 Mandel L, Surattanont F. Bilateral parotid swelling: a review. Oral Surg Oral Med Oral Pathol Oral Radiol Endod 2002;93:221-37.

3 Wilson KF, Meier JD, Ward PD. Salivary gland disorders. Am Fam Physician 2014;89:882-8.

4 Bialek EJ, Jakubowski W, Zajkowski P, et al. US of the major salivary glands: anatomy and spatial relationships, pathologic conditions, and pitfalls. Radiographics 2006;26:745-63.

5 Prasad RS. Parotid gland imaging. Otolaryngol Clin North Am 2016;49:285-312.

6 Mosier KM. Diagnostic radiographic imaging for salivary endoscopy. Otolaryngol Clin North Am 2009;42:949-72

7 Sumi M, Yamada T, Takagi Y, et al. MR imaging of labial glands. AJNR Am J Neuroradiol 2007;28:1552-6.

8 Sumi M, Takagi Y, Uetani M, et al. Diffusion-weighted echoplanar MR imaging of the salivary glands. AJR Am J Roentgenol 2002;178:959-65.

9 Becker M, Marchal F, Becker CD, et al. Sialolithiasis and salivary ductal stenosis: diagnostic accuracy of MR sialography with a three-dimensional extended-phase conjugate-symmetry rapid spin-echo sequence. Radiology 2000;217:347-58.

10 Yousem DM, Kraut MA, Chalian AA. Major salivary gland imaging. Radiology 2000;216:19-29.

11 Shah GV. MR imaging of salivary glands. Neuroimaging Clin N Am 2004; 14:777-808.

12 Armstrong MA, Turturro MA. Salivary gland emergencies. Emerg Med Clin North Am 2013;31:481-99.

13 Maroldi R, Farina D, Ravanelli M, et al. Emergency imaging assessment of deep neck space infections. Semin Ultrasound CT MR 2012;33:432-42.

14 Robson CD, Hazra R, Barnes PD, et al. Nontuberculous mycobacterial infection of the head and neck in immunocompetent children: CT and MR findings. AJNR Am J Neuroradiol 1999;20:1829-35.

15 Zenk J, Iro H, Klintworth N, et al. Diagnostic imaging in sialadenitis. Oral Maxillofac Surg Clin North Am 2009;21:275-92.

16 Gadodia A, Bhalla AS, Sharma R, et al. Bilateral parotid swelling: a radiological review. Dentomaxillofac Radiol 2011;40:403-14.

17 Madani G, Beale T. Inflammatory conditions of the salivary glands. Semin Ultrasound CT MR 2006;27:440-51.

18 Mandel L. Salivary gland disorders. Med Clin North Am 2014;98:1407-49.

19 Rubin P, Holt JF. Secretory sialography in diseases of the 
major salivary glands. Am J Roentgenol Radium Ther Nucl Med 1957;77:575-98.

20 Makula E, Pokorny G, Rajtár M, et al. Parotid gland ultrasonography as a diagnostic tool in primary Sjögren's syndrome. Br J Rheumatol 1996;35:972-7.

21 Koyama T, Ueda H, Togashi K, et al. Radiologic manifestations of sarcoidosis in various organs. Radiographics 2004;24:87-104.

22 Nwawka OK, Nadgir R, Fujita A, et al. Granulomatous disease in the head and neck: developing a differential diagnosis. Radiographics 2014;34:1240-56.

${ }^{23}$ Cheng SC, Wu VW, Kwong DL, et al. Assessment of postradiotherapy salivary glands. Br J Radiol 2011;84:393-402.

24 Ying M, Wu VW, Kwong DL. Comparison of sonographic appearance of normal and postradiotherapy parotid glands: a preliminary study. Ultrasound Med Biol 2007;33:1244-50.

25 Fujita A, Sakai O, Chapman MN, et al. IgG4-related disease of the head and neck: CT and MR imaging manifestations. Radiographics 2012;32:1945-58.

${ }^{26}$ Katabathina VS, Khalil S, Shin S, et al. Immunoglobulin G4related disease: recent advances in pathogenesis and imaging findings. Radiol Clin North Am 2016;54:535-51.
27 Capaccio P, Torretta S, Ottavian F, et al. Modern management of obstructive salivary diseases. Acta Otorhinolaryngol Ital 2007;27:161-72.

28 Terraz S, Poletti PA, Dulguerov P, et al. How reliable is sonography in the assessment of sialolithiasis? AJR Am J Roentgenol 2013;201:W104-9.

29 Gritzmann N, Rettenbacher T, Hollerweger A, et al. Sonography of the salivary glands. Eur Radiol 2003;13:964-75.

30 Andretta M, Tregnaghi A, Prosenikliev V, et al. Current opinions in sialolithiasis diagnosis and treatment. Acta Otorhinolaryngol Ital 2005;25:145-9.

31 Jain P, Jain R, Morton RP, et al. Plunging ranulas: high-resolution ultrasound for diagnosis and surgical management. Eur Radiol 2010;20:1442-9.

32 Adams A, Mankad K, Offiah C, et al. Branchial cleft anomalies: a pictorial review of embryological development and spectrum of imaging findings. Insights Imaging 2016;7:69-76.

33 Abrams AM, Melrose RJ, Howell FV. Necrotizing sialometaplasia. A disease simulating malignancy. Cancer 1973;32:130-5.

34 Farina D, Gavazzi E, Avigo C, et al. Case report. MRI findings of necrotizing sialometaplasia. Br J Radiol 2008;81:e173-5.

Received: September 15, 2016 - Accepted: December 12, 2016

Address for correspondence: Roberto Maroldi, Department of Radiology, University of Brescia, piazzale Spedali Civili 1, 25123 Brescia, Italy. Tel. +39 030 395900. Fax +39 030 3399897. E-mail: roberto.maroldi@unibs.it 